\title{
Qualitatively Comparing the Support Needs of People with Cancer Based on Their History of Anxiety/ Depression
}

\author{
Emma M. Richardson · Jennifer L. Scott • Natalie Schüz • \\ Kristy Sanderson · Benjamin Schüz
}

Received: February 15, 2017 / Published online: May 5, 2017

(C) The Author(s) 2017. This article is an open access publication

\begin{abstract}
Introduction: Research rarely considers the origin or history of a cancer patient's anxiety and/or depression, instead assuming that these illnesses are related to the cancer experience. The aim of this study was to compare differences in the support needs of people who have experienced anxiety/depression as part of the cancer experience and people who have not, as well as between people who have experienced episodic anxiety/depression and people who
\end{abstract}

Enhanced content To view enhanced content for this article go to http://www.medengine.com/Redeem/ 1B18F0607B30E54E.

Electronic supplementary material The online version of this article (doi:10.1007/s40487-017-0045-3) contains supplementary material, which is available to authorized users.

E. M. Richardson $(\varangle) \cdot$ J. L. Scott · B. Schüz Division of Psychology, School of Medicine, University of Tasmania, Hobart, Australia e-mail: Emma.Richardson@utas.edu.au

\section{N. Schüz}

School of Medicine, University of Tasmania, Hobart, Australia

K. Sanderson

School of Health Sciences, University of East Anglia, Norwich, England, UK have experienced long-term anxiety/ depression.

Methods: Twenty-one semi-structured interviews were conducted with people with a current or previous diagnosis of cancer, and a current or previous experience with anxiety and/or depression. Participants were split into four groups based on their history with cancer and anxiety/depression, and an inductive thematic analysis was conducted to identify themes across groups.

Results: Two superordinate themes (with three and two subordinate themes respectively) were found: 'coping with cancer' and 'health care system support provision'. Important differences were found across groups, with participants who had a history of anxiety/depression that was unrelated to their cancer diagnosis coping better with the combined burden of cancer and anxiety/depression, experiencing less fear of cancer recurrence, and highlighting more positive hospital and support service related experiences, than those whose anxiety/ depression was cancer related.

Conclusion: The origin and history of a person's anxiety/depression is important to consider when determining how they might cope with cancer, what their support needs are, and how much support they may require.

Keywords: Anxiety; Cancer; Co-morbidity; Depression; Needs; Support 


\section{INTRODUCTION}

Traditionally, psycho-oncology has examined anxiety and/or depression as conditions resulting from the cancer experience [1]. However, not all people with cancer develop anxiety/depression, and their levels of distress vary [2, 3]. Further, some people have pre-existing anxiety/ depression which then becomes co-morbid with cancer, while others experience episodes of anxiety/depression that occur in response to significant life events (including the cancer diagnosis). These examples highlight that anxiety/depression experienced by someone with cancer can have differential aetiology. However, the role of these origins and how they influence the cancer experience and the support needs of people with cancer and anxiety/depression is rarely considered in psycho-oncology research $[1,4]$.

The most important areas in need of support for people with cancer include physical health and daily living, psychological health, health system and information, and social support [5]. Although there are several interventions aimed at addressing these needs, a recent review has found that such interventions generally have limited effectiveness [6]. Further, though much of the research and interventions for people with cancer aim at improving distress, and one study has specifically examined how screening for distress might uncover unmet needs in people with cancer [7], research so far has not taken into account whether needs differ according to which illness (cancer or anxiety/ depression) came first, or the causes of anxiety/ depression. It is therefore unclear whether and how the needs of people with cancer are influenced by their history with anxiety/depression.

Comparing the support needs of people with varying histories of cancer and anxiety/depression will allow us to identify and discuss how the potentially differing needs of people according to their cancer and anxiety/depression history can best be addressed. For example, those who have experienced anxiety/depression as part of the cancer experience might differ from those who have not, and those who have experienced episodes of anxiety/depression in relation to significant life events might differ from those who have experienced long-term anxiety/depression. Support needs may differ between these groups, as long-term depression tends to be clinically more serious, is more often co-morbid with anxiety, requires more treatment, and leads to poorer social and psychological outcomes and reduced well-being, than episodic depression [8]. Examining differences between these groups is important because although intervention studies often take into account psychological distress at baseline [9], they rarely consider how a prior history of anxiety/depression may influence intervention effectiveness. Further, the outcomes of interventions for cancer patients with distress are often mixed, and previous systematic and meta-analytic reviews yielded disparate conclusions that are often difficult to interpret $[4,10,11]$. This highlights the need for more research on how the aetiology and sequence of cancer and mental illness may determine the support needs of people with cancer and anxiety/depression. This knowledge can form the basis for better targeted and more effective interventions, as well as improved access to appropriate support services, for people with cancer and anxiety/depression.

This study therefore aims to explicitly examine and compare the existing supports and needs of people with cancer and different histories of anxiety/depression (e.g., episodic versus long-term) through semi-structured interviews, allowing for insights to be gained through thematic analysis without researcher preconceptions. Two key questions were used to guide this study:

1. How do different histories of anxiety/depression influence the support needs of people with cancer?

2. How can support providers and health professionals better assist people with cancer and anxiety/depression based on their history with each illness?

\section{METHODS}

All procedures performed in studies involving human participants were in accordance with 
the ethical standards of the Tasmanian Social Science Human Research Ethics Committee (Reference Number: H0014664) and with the 1964 Helsinki declaration and its later amendments or comparable ethical standards. Informed consent was obtained from all individual participants included in the study.

\section{Participants}

Twenty-one participants were considered an appropriate sample size for this study based on information power [12]. Information power provides clear criteria for ascertaining appropriate sample sizes in qualitative research while addressing some of the limitations associated with the use of saturation $[12,13]$. The sample size for this study was ascertained based on its neither especially broad nor narrow aims, the inclusion of participants specific to the research aim (based on meeting inclusion criteria), strong rapport and interview dialogue between ER and participants (due to a strong knowledge of the theoretical background and previous experience working with cancer patients), and the use of cross-case analysis.

Participants were recruited via self-selection sampling through advertisements in Cancer Council Tasmania face-to-face support groups, local hospitals, and appropriate Facebook pages (cancer support groups). Persons interested in participating contacted the researcher via phone or email and were then invited to participate in the study upon meeting the following inclusion criteria: over 18 years of age, current or previous diagnosis with any type of cancer, current or previous experience with (diagnosed or treated for) anxiety or depression or both anxiety and depression.

\section{Materials}

Participants completed a consent form, demographics and illness characteristics questionnaire, and the shortened version of the Depression Anxiety Stress Scale (DASS-21; 14) to assess the symptom severity of each negative emotional state. An interviewer guide with open-ended questions was used to direct each interview (see Online Resource 1).

\section{Procedure}

Ethics approval was obtained from the Tasmanian Human Research Ethics Committee (H0014664). Following recruitment and meeting of recruitment criteria, participants were invited to participate in the study. Participants were informed of the purpose of the study, as well as the researchers' reasons for completing it (to increase understanding of cancer patients' thoughts and experiences with a view to improving support service provision) at this early stage. Relationships were generally not established prior to study commencement, though six participants were known to the interviewer through support work with Cancer Council Tasmania.

Face-to-face one-on-one semi-structured interviews were conducted following completion of the consent form, demographics and illness characteristics questionnaire, and DASS-21 [14]. The first author conducted each interview (ER; female PhD student with formal training and experience in support work with cancer patients) at a place convenient to participants (university, Cancer Council, or participants' homes). Audiotaped interviews lasted between 13 and $82 \mathrm{~min}(M=50 \mathrm{~min})$, ending after all key topics were covered. Field notes were not taken during interviews to maintain rapport and trust between interviewer and participant. Further, although participants were not offered a chance to review transcripts or provide feedback on identified themes, they were given an opportunity to request to be sent any research output or publications arising from the research.

\section{Analysis}

This study followed the COnsolidated criteria for REporting Qualitative research (COREQ) Checklist [15] (see Online Resource 2). Following verbatim transcription, transcripts were de-identified and imported into NVivo 10 [16] for data management and thematic analysis.

A subtle realist viewpoint guided analyses, where the researchers' subjective perceptions were acknowledged while attempting to 
represent the underlying existing reality under study [17]. An inductive thematic analysis was conducted [18]. This approach involves a data-driven way of identifying themes, where data is coded without any pre-existing coding frames or researcher preconceptions. Thematic analysis was guided by Braun and Clarke [18], who provide clear steps for conducting such an analysis.

Data analysis began with data immersion (ER transcribing interviews and reading completed transcripts several times to familiarise with the data). All transcripts were then coded into units of meaning by ER using NVivo 10. Units of meaning were used for coding, as predefined blocks of text (lines or sentences) may inaccurately reflect intended meanings of participants and important contextual information may be missed [19]. A second coder $(\mathrm{JH})$ coded 5/21 (23.81\%) interviews to ensure reliability. Between ER and JH inter-rater agreement was high $(92.25 \%)$ and inter-rater reliability was good (Cohen's $\kappa=0.664$ ).

Based on their history with cancer and anxiety/depression, participants were categorised by two authors (ER and JS) into four groups: cancer associated with anxiety/depression AND a history of episodic anxiety/depression or anxiety/depression caused by cancer alone (Group 1; ten participants); cancer associated with anxiety/depression AND long-term anxiety/depression (Group 2; six participants); cancer that was not associated with anxiety/ depression AND a history of episodic anxiety/ depression (Group 3; one participant); and

Table 1 Participant groupings based on history of cancer and anxiety/depression

\begin{tabular}{lll}
\hline & $\begin{array}{l}\text { Episodic } \\
\text { anxiety/ } \\
\text { depression (or } \\
\text { caused by cancer } \\
\text { alone) }\end{array}$ & $\begin{array}{l}\text { Long-term } \\
\text { anxiety/ } \\
\text { depression }\end{array}$ \\
\hline $\begin{array}{c}\text { Cancer associated } \\
\text { anxiety/depression }\end{array}$ & Group 1 & Group 2 \\
$\begin{array}{c}\text { No cancer associated } \\
\text { anxiety/depression }\end{array}$ & Group 3 & Group 4 \\
\hline
\end{tabular}

cancer that was not associated with anxiety/ depression AND long-term anxiety/depression (Group 4; four participants; see Table 1). Following the creation of these groups, codes were collated into potential themes, with themes then reviewed, cross-checked for overlap, defined, and named.

\section{RESULTS}

\section{Demographics and Illness Characteristics}

Twenty-two participants were seen, with 21 (three males, 18 females) meeting inclusion criteria for participation (one participant had no formal diagnosis of or treatment for anxiety/ depression). Participants were aged between 23 and 75 years $(M=50$ years, $S D=18$ years $)$ and had a current or previous cancer diagnosis, with four participants experiencing anxiety (19.05\%), five experiencing depression (23.81\%), and 12 participants experiencing both anxiety and depression at some point throughout their lifespan $(57.14 \%)$. The majority of participants $(16,76.19 \%)$ were born in Australia, had completed year 12 or above (18, $85.71 \%$ ), were employed (six, $28.57 \%$ ), retired (six, 28.57\%), or not working due to health (six, $28.57 \%$ ), and were married (ten, $47.62 \%$ ), in a de-facto relationship (four, 19.05\%), or single (five, $23.81 \%$ ). Participants primary cancer diagnoses included breast (nine, $42.86 \%$ ), bowel (five, 23.81\%), sarcoma (one, 4.76\%), Hodgkin's lymphoma (one, 4.76\%), non-Hodgkin's lymphoma (one, $4.76 \%$ ), brain (one, $4.76 \%$ ), liver (one, $4.76 \%$ ), prostate (one, $4.76 \%$ ), and unknown primary (one, $4.76 \%$ ). Three participants had received a second primary cancer diagnosis $(14.29 \%)$, four had received a diagnosis of secondary/metastatic cancer (19.05\%), 16 were post-treatment $(76.19 \%)$, and five were peri-treatment $(23.81 \%)$. Participants cancer treatments included surgery $(20,95.24 \%)$, chemotherapy $(16,76.19 \%)$, radiotherapy $(12$, $57.14 \%$ ), hormone therapy (six, 28.57\%), and tablet medication (seven, 33.33\%). Nine participants had no other multimorbid conditions $(42.86 \%)$, nine had one other multimorbid condition $(42.86 \%)$, and three had two other 
multimorbid conditions (14.29\%). These demographics and illness characteristics are available in table form in Online Resource 3.

\section{DASS-21}

On average, anxiety scores were in the moderate range $(M=7.43, S D=8.80)$, stress scores were in the severe range $(M=15.52, S D=8.02)$, and depression scores were in the extremely severe range $(M=14.00, S D=11.33)$, though scores on each subscale ranged from normal to extremely severe.

\section{Thematic Analysis}

Differences between the four participant groups (see Table 1) were identified across two superordinate themes, with each superordinate theme broken into several additional subordinate themes (see Fig. 1). The first superordinate theme, 'Coping with Cancer', describes and compares coping strategies, personal relationships, and fear of cancer recurrence (FCR) across participant groups. The second superordinate theme, 'Health Care System Support Provision', describes and compares hospital care and support services across participant groups. To illustrate these key themes and highlight important issues, quotations will be presented. Pseudo-names have been used to preserve the anonymity of participants.

\section{Coping with Cancer}

In general, participants in Groups 1 and 2 did not cope as well with their cancer as participants in Groups 3 and 4. More specifically, participants in Groups 1 and 2 required more coping strategies and resources to cope with their cancer, while also perceiving and describing less social support and a higher FCR, than participants in Groups 3 and 4.

Coping Strategies Most participants in Groups 1 and 2 received formal psychological treatment for cancer-related anxiety/depression, though participants in Group 2 generally described this treatment in much less detail and/or were less likely to include this as a coping strategy for cancer. Participants in Groups 3 and 4 reported the use of less formal coping and self-management strategies, such as being positive, taking control where possible, keeping busy, and keeping life as normal as possible, with no treatment for cancer-related anxiety and depression described by these participants.

I couldn't exercise and stuff which I'd previously done a lot. ... I tried to, you

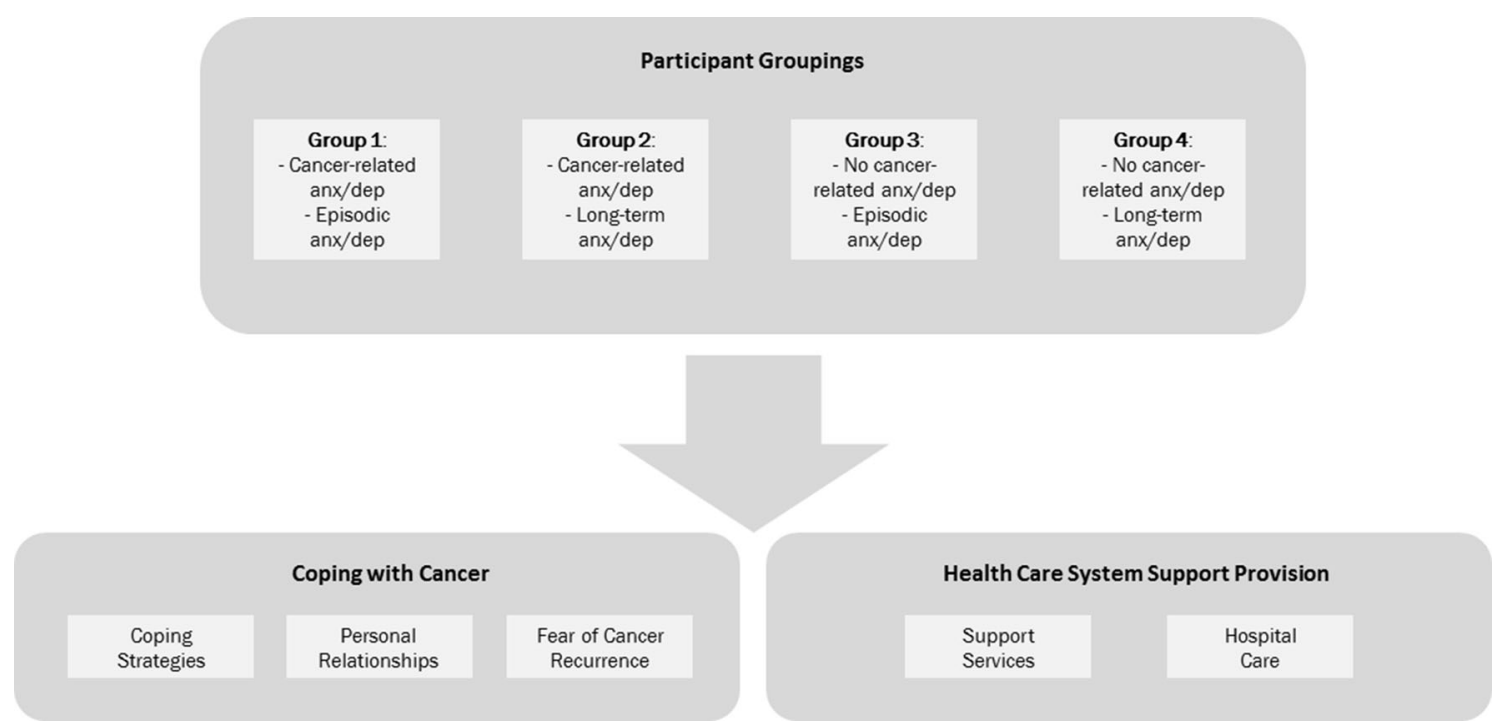

Fig. 1 Thematic map comparing support needs of people with cancer and anxiety/depression 
know sort of, walk and stuff rather than running, which I couldn't do a whole lot, but I could do some. Um, and a lot of it was just getting support from other people as well. (ER: Mmhm.) You know, friends and family and that kind of stuff. Um, and just, like a big thing for me was not thinking about it constantly. So doing, keeping my mind busy. (Dominic, Group 4)

With regard to support groups specifically, eight of ten people in Group 1, and four out of five people in Group 2, attended a support group for cancer-related support. The participants in Groups 3 and 4 did not attend a cancer-related support group. The majority of participants attending support groups found them highly beneficial.

Personal Relationships For participants in Group 1, strong social support was important, with participants describing how their cancer diagnosis strengthened some relationships but led to the loss of others (most often friendships). Cancer was sometimes described as changing the individual, and therefore changing their relationships. Although some participants in Group 2 described strengthened relationships with their partner or spouse, the majority of these participants described losing friendships and feeling isolated, with less positive social support mentioned overall.

I had a ... friend the other day, a month or two ago I sent her a message and she asked me how I was and I just text her back saying I felt like crap and I was angry and everything, and you know you don't hear from them for a month. So you've got to be careful about what you say otherwise... (ER: Mm.) people just walk away or they just stay away for a, you know, yeah. (Rosalina, Group 2)

In contrast, participants in Groups 3 and 4 described strong social support and personal relationships, with no loss of relationships or support from loved ones described.

Fear of Cancer Recurrence (FCR) FCR was experienced by participants in Groups 1 and
2, particularly when waiting for test results and when experiencing physiological symptoms that could be a sign of recurrence. However, only participants in Group 1 experienced highly maladaptive FCR post cancer treatment, which was described as occurring once they had time to process what had happened to them.

It wasn't until after, well after 12 months that I really ... started to feel the effects of anxiety and stuff. (ER: Mm.). When the oncologist visits dropped off, when you didn't need to see a surgeon again. ... When chemo finished ... So that's when it started to mess with me. (Freya, Group 1)

If I started to feel an ache or pain somewhere ... I would get that focused on it I'd physically make, make myself ... sick, and I'd, you know, I'd feel worse than I was, you know, I'd have tingly feelings and all sorts of weird feelings that I thought "well I'm going to die" or something... (Eve, Group 1).

Participants in Groups 3 and 4 were much less concerned about FCR, with minor worry occurring when awaiting test results and when thinking about the possibility that they may miss symptoms of a recurrence.

\section{Health Care System Support Provision}

In general, participants in Groups 2 and 4 described experiencing good support from the hospital and appropriate integration with support services, while participants in Group 1 described a lack of support and information from the hospital and a lack of integration with appropriate support services. The participant in Group 3 made no specific comments regarding the hospital system or use of support services.

Hospital Care Participants in Group 1 generally described a lack of support and information from the hospital with regard to mental health and support services (though some participants felt adequately supported). This was particularly highlighted by participants at the post-treatment stage. 
It's a business ... getting the cancer sorted out, and getting the cancer over with, as far as the hospital and all that is concerned. (ER: Mm.). So, and it was like, once it stopped it was like "ah, what now?" ... I said to my psychologist today, "It was like... the treatment should only be starting now..." (Sienna, Group 1)

In contrast, participants in Groups 2 and 4 felt that the hospital system was mostly good, with less needs described.

I went Private, and they were brilliant. ... They'd give you this and they'd give you that, and I didn't take any of it, because well why, there was people going to be worse off than me. (Quinn, Group 4)

In general, interviews suggested that those with no prior support or treatment in place for anxiety/depression required more support from the hospital than those with long-term and/or previous mental illness experiences.

Support Services A lack of integration with appropriate support services for people with cancer, a lack of information, and few links with counselling or psychological support services, were described by multiple participants in Group 1. In particular, doctors (and the hospital system) were often described as failing to link their patients with appropriate support services.

I don't know what it would be like for anybody who, um, doesn't have support. ... cause the Doctor didn't, nobody sort of said "do you want counselling?". ... there was no offer of counselling. ... Even nobody mentioned the Support Group. (Bella, Group 1)

Doctors need to be more responsible, more proactive... and stop being this sausage factory of putting people through as quickly as you possible. ... And I think a lot of people probably find it more difficult after treatment has finished, because there's nothing. ... And ... the lack of links with people that are integrative medicine specialists, like um homoeopathic, ah naturopaths, physio ... psychologists.
There's just no central link to the whole thing. (Bianca, Group 1)

In contrast, participants in Group 2 (bar one) and Group 4 perceived solid links with support services, though some of these participants had links with mental health services already in place prior to their cancer diagnosis.

\section{DISCUSSION}

This qualitative study aimed to compare and examine the existing supports and needs of people with cancer and different histories of depression/anxiety. Our findings suggest that a person's support needs may differ based on their history of coping with anxiety/depression premorbid to their cancer diagnosis. More specifically, we found that participants with anxiety/depression associated with cancer (no matter the origin or history of their anxiety/ depression) were much more likely to access formal coping strategies such as psychological treatment for cancer-related anxiety/depression, attend cancer support groups, lose personal relationships because of their cancer, and experience severe maladaptive FCR, than participants with anxiety/depression not associated with cancer (no matter the origin or history of their anxiety/depression). Our results also found that participants with episodic or cancer-related anxiety/depression felt less well supported by the hospital system and experienced less links with support services than participants with long-term anxiety/depression.

Many of the participants in this study had a vulnerability for experiencing anxiety/depression associated with their cancer due to their history of long-term anxiety/depression [20]. Interestingly, however, only some of these participants experienced cancer-related anxiety/ depression (Group 2), with these same participants also demonstrating poorer coping, needing more anxiety/depression treatment, and experiencing worse FCR. To better understand why this might have occurred, it is important to consider how and why those with a premorbid history of long-term anxiety/depression and anxiety/depression in response to their cancer 
(Group 2) might differ from those with a similar history who did not experience any cancer-related anxiety/depression (Group 4). Participants in Group 2 were generally younger than those in Group 4, had an average of one additional chronic illness per person (whereas only one participant in Group 4 had an additional chronic illness), reported they received more misinformation and misdiagnoses from health professionals, described less positive social support interactions, and perceived more, and more severe, cancer-related psychosocial consequences. This is in line with previous research that suggests that cancer patients who are younger [21-23], have additional chronic illnesses [24, 25], less social support [26, 27], and more negative illness representations [28], are more likely to experience higher levels of distress. This suggests that together with these potential risk factors, people who also have a history of long-term anxiety/depression and who have not developed coping strategies to manage these issues might be at risk to develop cancer-related anxiety/ depression, and therefore may be more at need for support to prevent or decrease such anxiety/depression.

Participants with episodic or cancer-related anxiety/depression (Group 1) often felt less supported by the hospital system and experienced less access to appropriate support and mental health services than participants with long-term anxiety/depression (Groups 2 and 4). Our findings suggest that this was likely due to a lack of prior exposure to mental health and support services, as participants with previous or existing links with such services required less hospital support and service access. One other potential explanation for this pattern of results lies with the setting for our study, a regional area of Australia [29] with no cancer-specific hospitals, cancer centres, and a lack of individualised services and tailored support (particularly psychological services). Research suggests that people in rural/regional settings often experience worse outcomes, limited access to tailored cancer-specific hospital care, a lack of psychological support, and few links and referrals to other key support services $[30,31]$.

\section{Implications}

Our study suggests that a person's history of anxiety/depression may influence how they cope with their cancer experience. In terms of distress trajectories in cancer patients $[2,3,21,32]$, our findings suggest that those who have a history of anxiety/depression that is unrelated to cancer might be better able to cope with the combined burden resulting from both the mental and physical illness than those whose anxiety/depression is related to cancer. The knowledge that inexperience in coping with anxiety/depression might place a person at risk of a trajectory of chronic distress post cancer could inform targeted implementation of appropriate resources and services for people at risk. However, more research is needed to determine which facets of a person's anxiety/ depression history might be most important in predicting such trajectories. Coping repertoire and skills to manage psychological distress would be particularly worthy of exploration.

In terms of service provision, our study is in line with previous research $[31,33]$ and suggests a need for patients to receive additional support and information from hospitals, as well as referrals to external mental health and support services where required. However, hospitals often lack the resources to implement such changes, particularly in rural/regional areas. This study suggests that these limited resources are best directed towards increasing information and links with support services for people with cancer-related anxiety/depression, rather than for those who report coping well with a history of long-term anxiety/depression. This is because the latter group often already have existing support systems in place, while people with cancer related anxiety/depression are distressed, have less social support, and experience more severe FCR. For the same reasons it is also suggested that health professionals recommend the use of appropriate support services (including support groups) to people with cancer-related anxiety/depression. Our findings highlight the importance of investigating a cancer patient's history of anxiety/depression to determine how much support they may (or may not) require. 


\section{Limitations}

Although we attempted to differentiate between people with episodic anxiety/depression and people with long-term anxiety/depression, no clinical diagnostic measures or criteria were used to assess this during the interview process. Instead, two researchers (ER and JS-a clinical psychologist) placed participants into groups based on participant data (demographic and interview). Participants were assigned to groups based upon consideration of their reported family history of psychological disorders, psychological responses to past significant life events and premorbid experience of anxiety/depression generally, as well as their reports of their response to their cancer experience.

Due to the widely varied cancer types, cancer stages, and treatment types, experienced by our sample, more consensus could potentially have been found with the use of a more focused sample, such as the inclusion of participants who only have bowel cancer or early-stage disease (Stage 1). Conversely, a more diverse sample may have led to additional insights. As our sample provided both overlapping and unique representations across groups, a good balance between specificity and diversity appears to have been achieved. However, as only one participant had episodic anxiety/depression that was not related to cancer (Group 3), results from this specific participant category should not be generalised to others in similar situations. Furthermore, given its small sample size and exploratory nature, caution must be taken before generalising any result from this study.

\section{CONCLUSIONS}

In conclusion, our study has highlighted the need for both researchers and health professionals to give more consideration to the origin and history of a person's anxiety/depression in order to determine how they might cope with cancer, their support needs, and the amount of support they may require.

\section{ACKNOWLEDGEMENTS}

The authors wish to gratefully acknowledge Jane Hyde for coding a subset of interview transcripts. No funding or sponsorship was received for this study or publication of this article. All named authors meet the International Committee of Medical Journal Editors (ICMJE) criteria for authorship for this manuscript, take responsibility for the integrity of the work as a whole, and have given final approval for the version to be published.

Disclosures. Emma M. Richardson, Jennifer L. Scott, Natalie Schüz, Kristy Sanderson, and Benjamin Schüz have nothing to disclose.

Compliance with Ethics Guidelines. All procedures performed in studies involving human participants were in accordance with the ethical standards of the Tasmanian Social Science Human Research Ethics Committee (Reference Number: H0014664) and with the 1964 Helsinki Declaration and its later amendments or comparable ethical standards. Informed consent was obtained from all individual participants included in the study.

Data Availability. The datasets during and/ or analysed during the current study are available from the corresponding author on reasonable request.

Open Access. This article is distributed under the terms of the Creative Commons Attribution-NonCommercial 4.0 International License (http://creativecommons.org/licenses/ by-nc/4.0/), which permits any noncommercial use, distribution, and reproduction in any medium, provided you give appropriate credit to the original author(s) and the source, provide a link to the Creative Commons license, and indicate if changes were made.

\section{REFERENCES}

1. Pasquini M, Biondi M. Depression in cancer patients: a critical review. Clin Pract Epidemiol 
Ment Health. 2007;3:1-9. doi:10.1186/1745-01793-2.

2. Helgeson VS, Snyder P, Seltman H. Psychological and physical adjustment to breast cancer over 4 years: identifying distinct trajectories of change. Health Psychol. 2004;23:3-15. doi:10.1037/02786133.23.1.3.

3. Henselmans I, Helgeson VS, Seltman H, de Vries J, Sanderman R, Ranchor AV. Identification and prediction of distress trajectories in the first year after a breast cancer diagnosis. Health Psychol. 2010;29:160-8. doi:10.1037/a0017806.

4. Jacobsen PB, Jim HS. Psychosocial interventions for anxiety and depression in adult cancer patients: achievements and challenges. CA Cancer J Clin. 2008;58:214-30. doi:10.3322/CA.2008.0003.

5. Harrison JD, Young JM, Price MA, Butow PN, Solomon MJ. What are the unmet supportive care needs of people with cancer? A systematic review. Support Care Cancer. 2009;17:1117-28. doi:10.1007/ s00520-009-0615-5.

6. Carey M, Lambert S, Smits R, Paul C, Sanson-Fisher $\mathrm{R}$, Clinton-McHarg T. The unfulfilled promise: a systematic review of interventions to reduce the unmet supportive care needs of cancer patients. Support Care Cancer. 2012;20:207-19. doi:10.1007/ s00520-011-1327-1.

7. van Scheppingen C, Schroevers MJ, Smink A, van der Linden YM, Mul VE, Langendijk JA, Coyne JC, Sanderman R. Does screening for distress efficiently uncover meetable unmet needs in cancer patients? Psycho-Oncology. 2011;20:655-63. doi:10.1002/ pon.1939.

8. Angst J, Gamma A, Rössler W, Ajdacic V, Klein DN. Long-term depression versus episodic major depression: results from the prospective Zurich study of a community sample. J Affect Disord. 2009;115:112-21. doi:10.1016/j.jad.2008.09.023.

9. Schneider S, Moyer A, Knapp-Oliver S, Sohl S, Cannella D, Targhetta V. Pre-intervention distress moderates the efficacy of psychosocial treatment for cancer patients: a meta-analysis. J Behav Med. 2010;33:1-14. doi:10.1007/s10865-009-9227-2.

10. Lepore SJ, Coyne JC. Psychological interventions for distress in cancer patients: a review of reviews. Ann Behav Med. 2006;32:85-92. doi:10.1207/ s15324796abm3202_2.

11. Galway K, Black A, Cantwell M, Cardwell CR, Mills M, Donnelly M. Psychosocial interventions to improve quality of life and emotional wellbeing for recently diagnosed cancer patients. Cochrane
Database Syst Rev. 2012;11:CD007064. doi:10. 1002/14651858.CD007064.pub2.

12. Malterud K, Siersma VD, Guassora AD. Sample size in qualitative interview studies: guided by information power. Qual Health Res. 2015;26:1753-60. doi:10.1177/1049732315617444.

13. Glaser BG, Strauss AL. The discovery of grounded theory: strategies for qualitative research. New Bruswick: Aldine Transaction; 2009.

14. Lovibond SH, Lovibond PF. Psychology foundation of Australia: manual for the depression anxiety stress scales. 2nd ed. Sydney: Psychology Foundation of Australia; 1995.

15. Tong A, Sainsbury P, Craig J. Consolidated criteria for reporting qualitative research (COREQ): a 32-item checklist for interviews and focus groups. Int J Qual Health Care. 2007;19:349-57. doi:10.1093/ intqhc/mzm042.

16. QSR International Pty Ltd. NVivo qualitative data analysis Software (Version 10). 2014.

17. Mays N, Pope C. Assessing quality in qualitative research. BMJ. 2000;320:50-2. doi:10.1136/bmj. 320.7226.50.

18. Braun V. Clarke V using thematic analysis in psychology. Qual Res Psychol. 2006;3:77-101. doi:10. 1191/1478088706qp063oa.

19. Campbell JL, Quincy C, Osserman J, Pedersen OK. Coding in-depth semistructured interviews: problems of unitization and intercoder reliability and agreement. Sociol Methods Res. 2013;42:294-320. doi: $10.1177 / 0049124113500475$.

20. Mehta RD, Roth AJ. Psychiatric considerations in the oncology setting. CA Cancer J Clin. 2015;65:299-314. doi:10.3322/caac.21285.

21. Dunn J, Ng SK, Holland J, Aitken J, Youl P, Baade PD, Chambers SK. Trajectories of psychological distress after colorectal cancer. Psycho-Oncology. 2013;22:1759-65. doi:10.1002/pon.3210.

22. Hulbert-Williams N, Neal R, Morrison V, Hood K, Wilkinson C. Anxiety, depression and quality of life after cancer diagnosis: what psychosocial variables best predict how patients adjust? Psycho-Oncology. 2012;21:857-67. doi:10.1002/pon.1980.

23. Linden W, Vodermaier A, Mackenzie R, Greig D. Anxiety and depression after cancer diagnosis: prevalence rates by cancer type, gender, and age. J Affect Disord. 2012;141:343-51. doi:10.1016/j.jad. 2012.03.025. 
24. Ritchie CS, Kvale E, Fisch MJ. Multimorbidity: an issue of growing importance for oncologists. J Oncol Pract. 2011;7:371-4. doi:10.1200/JOP.2011. 000460.

25. Gunn JM, Ayton DR, Densley K, Pallant JF, Chondros P, Herrman HE, Dowrick CF. The association between chronic illness, multimorbidity and depressive symptoms in an Australian primary care cohort. Soc Psychiatry Psychiatr Epidemiol. 2010;47:175-84. doi:10.1007/s00127-010-0330-z.

26. Pinar G, Okdem S, Buyukgonenc L, Ayhan A. The relationship between social support and the level of anxiety, depression, and quality of life of Turkish women with gynecologic cancer. Cancer Nurs. 2012;35:229-35. doi:10.1097/NCC.0b013e31822c4 $7 \mathrm{bd}$.

27. Singer S, Krauß O, Keszte J, Siegl G, Papsdorf K, Severi E, Hauss J, Briest S, Dietz A, Brähler E. Predictors of emotional distress in patients with head and neck cancer. Head Neck. 2012;34:180-7. doi:10.1002/hed.21702.

28. Richardson EM, Schüz N, Sanderson K, Scott JL, Schüz B. Illness representations, coping, and illness outcomes in people with cancer: a systematic review and meta-analysis. Psycho-Oncology. 2016; doi:10.1002/pon.4213.
29. Australian Bureau of Statistics. Australian statistical geography standard (ASGS): volume 5-remoteness structure. 2011. 1270.0.55.005.

30. Butow PN, Phillips F, Schweder J, White K, Underhill C, Goldstein D. Psychosocial well-being and supportive care needs of cancer patients living in urban and rural/regional areas: a systematic review. Support Care Cancer. 2012;20:1-22. doi:10.1007/ s00520-011-1270-1.

31. Gunn K, Turnbull D, McWha JL, Davies M, Olver I. Psychosocial service use: a qualitative exploration from the perspective of rural Australian cancer patients. Support Care Cancer. 2013;21:2547-55. doi:10.1007/s00520-013-1812-9.

32. Lam WWT, Shing YT, Bonanno GA, Mancini AD, Fielding R. Distress trajectories at the first year diagnosis of breast cancer in relation to 6 years survivorship. Psycho-Oncology. 2012;21:90-9. doi:10.1002/pon.1876.

33. Rainbird K, Perkins J, Sanson-Fisher R, Rolfe I, Anseline $\mathrm{P}$. The needs of patients with advanced, incurable cancer. Br J Cancer. 2009;101:759-64. doi:10.1038/sj.bjc. 6605235 . 